\title{
Vulnerabilidade à seca e (i)mobilidade no Nordeste brasileiro: partir ou resistir?
}

\author{
Vulnerability at drought and (im)mobility in the Brazilian \\ Northeast: leave or resist?
}

Isac Alves Correia ${ }^{\mathrm{a}}$

Alisson Flávio Barbierib

${ }^{a}$ Doutorando em Demografia, Universidade Federal de Minas Gerais,

Belo Horizonte, MG, Brasil

E-mail: isc.correia49@gmail.com

${ }^{b}$ Professor Adjunto do Departamento de Demografia, Universidade Federal de Minas Gerais, Belo Horizonte, MG, Brasil. E-mail: barbieri@cedeplar.ufmg.br

doi:10.18472/SustDeb.v10n2.2019.19806

Received: 30/11/2018

Accepted: 14/08/2019

ARTICLE - VARIA

\section{RESUMO}

Baseado em um survey com 1.064 domicílios do Seridó potiguar, região do semiárido nordestino, este trabalho discute como os indivíduos ajustam respostas de mobilidade ao perceberem os impactos das estiagens sobre os meios de subsistência. Nossos resultados mostram respostas usualmente de curto prazo envolvendo mobilidade cotidiana ou sazonal em relação à mudança permanente de residência. Também foi possível observar maior participação feminina nesses tipos de mobilidade, o que indica associação com o seu caráter de curta distância. Os programas sociais mostraram grande relevância nesse processo, com a possibilidade de duplo efeito: apoiando famílias sem emigrantes ou subsidiando a emigração de pelo menos um morador. Por fim, conclui-se que a migração e/ou a oferta de trabalho nos espaços urbanos foram ferramentas importantes para as famílias lidarem com o risco ambiental, o que requer instituições fortalecidas para gerenciar os potenciais impactos negativos para as regiões de destino desses indivíduos.

Palavras-chave: Secas; Mobilidade; Mudanças Ambientais; Riscos; Semiárido do Nordeste Brasileiro.

\section{ABSTRACT}

Based on a survey with 1,064 households in the Seridó Potiguar, in the Brazilian northeastern Semiarid region, this paper discusses how individuals adjust mobility responses when they perceive the impacts of droughts on their livelihoods. Our results show that short-term responses are more common with daily or seasonal mobility in relation to permanent residence change. We also observe a greater female participation in these types of mobility, probably due to its shorter distance. Cash transfer programs show great relevance in this process, supporting families without emigrants or subsidizing the emigration of at least one resident. Finally, we conclude that migration and/or labor supply in urban areas have been important strategies for families to deal with the risk, which requires strong institutions to manage the potential negative impacts on the destination regions of these individuals.

Keywords: Droughts; Mobility; Environmental Changes; Risks; Brazilian Northeast Semiarid Region. 


\section{INTRODUÇÃO}

No Nordeste brasileiro viviam em 2010 cerca de 53 milhões de habitantes, o equivalente a $27,8 \%$ da população brasileira (IBGE, 2010). Essa região é marcada por seu processo histórico de injustiças sociais desde sua colonização, com a distribuição da terra de forma desigual. As estiagens severas e prolongadas, que tendem a se agravar com as mudanças climáticas, elevam as desigualdades na região que já são superiores às demais do País (GONÇALVES, 2001).

O Nordeste tem experimentado ao longo das últimas décadas um processo de urbanização ${ }^{1}$ mais acelerado em relação às demais regiões do País, embora ainda seja a região que menos concentre população na zona urbana (73\%). Nela está a maior parte dos municípios do semiárido, que apresentam índices pluviométricos abaixo de 800 mm e precipitação praticamente três vezes superior a isso (OJIMA, 2015).

Não obstante, o Nordeste não é uma região homogênea em se tratando das características do processo de urbanização. Assim como os investimentos, serviços de saúde e educação, e oportunidades de empregos diversos, boa parte da população está concentrada nos centros urbanos das grandes cidades e nas regiões metropolitanas, tais como Fortaleza, Recife e Salvador. Essas áreas não estão imunes aos efeitos de secas extremas e muito menos dos impactos das mudanças climáticas - sobretudo porque boa parte dessas cidades está localizada em zonas costeiras - como o potencial aumento futuro do nível do mar e a elevação da temperatura média, conforme indicam os relatórios do Painel Intergovernamental sobre Mudanças Climáticas (IPCC) (IPCC, 2012, 2013, 2014).

Ao mesmo tempo em que exige maiores esforços das grandes cidades, a mobilidade pode acentuar processos de degradação em áreas rurais. Além disso, situações específicas de vulnerabilidade podem ser intensificadas pela mobilidade (ADGER et al., 2015). As respostas de mobilidade às secas, além de serem estreitamente ligadas a determinadas situações de vulnerabilidade, apresentam desafios institucionais importantes (CORREIA; OJIMA, 2017).

Nesse sentido, o artigo discute como os indivíduos ajustam suas respostas de mobilidade ao perceberem os impactos das estiagens sobre os meios de subsistência e as implicações institucionais, como as políticas de proteção social e o planejamento urbano. Nós utilizamos o caso da população do Seridó potiguar (Figura 1), uma região localizada no semiárido nordestino e que possui mais de 216 mil habitantes (IBGE, 2010). A fonte de dados é originária de um survey realizado com mais de mil domicílios urbanos. No Seridó predominam altas temperaturas, baixa fertilidade do solo e regime de chuvas irregulares (BARBIERI et al., 2018; CORREIA; OJIMA, 2018).

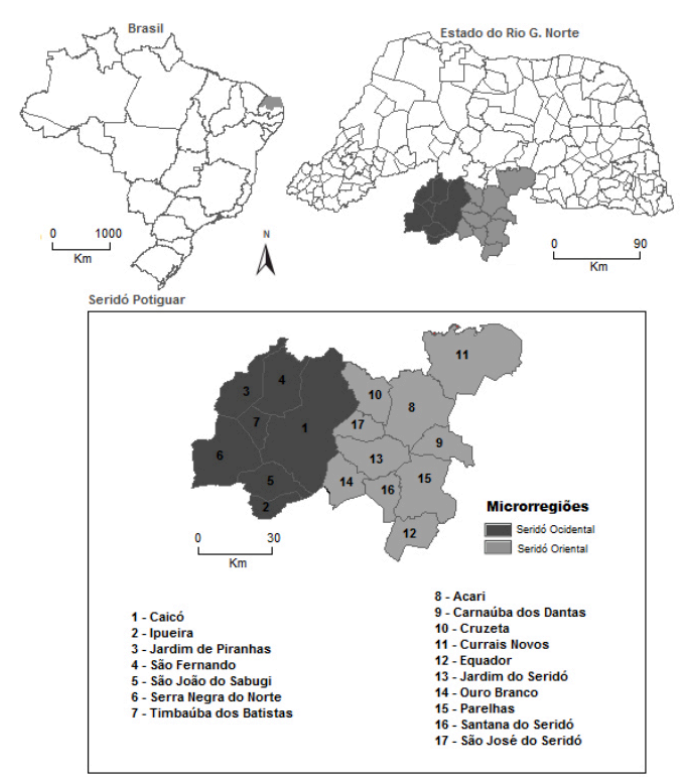

Figura 1 | Localização do Seridó potiguar, estado do Rio Grande do Norte e municípios inseridos nas microrregiões do Seridó Ocidental e do Seridó Oriental - 2010. 
Argumentamos que a região do Seridó é extremamente relevante para discutir a relação entre as respostas de mobilidade dos indivíduos e a seca no semiárido pelo fato de apresentar altas temperaturas, baixa fertilidade do solo e regime de chuvas irregular, além de uma forte dependência da população a atividades agropastoris. Além disso, o semiárido em particular é uma região em que o fator institucional representado pelos programas de transferência de renda possui um forte impacto sobre as estratégias familiares de subsistência e adaptação à seca (CORREIA; OJIMA, 2018).

A região enfrentou entre 2011-2012 uma das maiores estiagens dos últimos 30 anos e a maior crise hídrica dos últimos 50 anos, seca que se prolongou até o ano de realização da coleta de dados desta pesquisa (2017). Outro fator importante é que mais de $84 \%$ da população vive na zona urbana, sendo tal característica útil para entender sinergias entre o rural e o urbano em relação às estratégias domiciliares.

\section{MOBILIDADE E ESTRATÉGIAS DE ADAPTAÇÃO NO SEMIÁRIDO}

A Região Nordeste é historicamente conhecida por sua vulnerabilidade socioeconômica e ambiental que, notadamente, tem relação com suas características morfoclimáticas, o que tem motivado discussões teóricas importantes (AB'SABER, 1999; ARAÚJO, 2012; CASTRO, 2001; FURTADO, 1959; MARTINE, 1994).

Por outro lado, e reforçando a natureza socioambiental da vulnerabilidade na região, às questões climáticas somam-se, como dimensão da vulnerabilidade, discussões sobre o processo histórico de construção e reprodução de injustiças e desigualdades sociais na região desde a época da colonização do País (GONÇALVES, 2001).

Durante décadas a literatura regional se apropriou do fenômeno da seca enquanto elemento de destaque para explicação das condições sociais do Nordeste. Embora esse esforço tenha explicado o processo de usurpação política e econômica, as intervenções políticas que escapam de formas de preservar essa dependência da população diante do discurso da seca praticamente inexistem no cenário atual (OJIMA, 2015).

Concomitantemente, o Nordeste como um todo é classificado como uma região expulsora de população seja por fatores ambientais, incluindo a seca, seja por indicadores socioeconômicos e/ou regionais inferiores às áreas de atração dessas populações (FUSCO; DUARTE, 2010). No entanto, em nenhuma dessas discussões as questões ambientais são tratadas como centrais (OJIMA, 2015).

Considerando as mudanças nos fluxos migratórios observadas a partir da década de 1990 (CUNHA, 2005), os fluxos inter-regionais passam a ser observados partindo de uma visão mais holística, e peculiaridades são assumidas especialmente para aqueles da Região Nordeste. Entre essas peculiaridades, ganham destaque as políticas de desenvolvimento regional que, embora sejam importantes para entender os fluxos migratórios, ainda são incipientes para o contexto do semiárido (OJIMA, 2015).

Há evidências de que com as mudanças climáticas os eventos extremos, como as secas, serão acentuados, intensificando os fluxos migratórios (BARBIERI et al., 2010; IPCC, 2014; MYERS, 2002) e reconfigurando as situações de vulnerabilidade no espaço geográfico (ADGER et al., 2015). Esse debate se tornou mais caloroso e perene sobretudo a partir da publicação dos relatórios do Painel Intergovernamental para Mudanças Climáticas (IPCC).

Por outro lado, não há um consenso na literatura tanto na discussão se a migração seria uma alternativa de adaptação, ou se esta contribuiria para reiterar cenários de vulnerabilidade (BARBIERI, 2011) quanto no que diz respeito à intensificação desses movimentos populacionais motivados por mudanças ambientais (ADGER et al., 2015; KOUBI; STOLL; SPILKER, 2016).

Para o semiárido brasileiro, essa relação entre emigração e mudanças ambientais é praticamente um consenso. Não obstante existem lacunas que desafiam essa relação. De acordo com Ojima (2015), essas situações de vulnerabilidade no Nordeste, identificadas historicamente na dinâmica populaçãoambiente, poucas vezes foram exploradas na literatura devido ao cenário econômico. Tal afirmação se justifica pela dificuldade em captar, pelas fontes estatísticas oficiais, diferentes formas de mobilidade e de combinações de motivações (BARBIERI, 2011). 
Ainda que o estigma da seca se comporte como um poderoso fator de expulsão da população nordestina acentuando a migração forçada, os diferenciais socioeconômicos das regiões receptoras, quando confrontadas com as oportunidades e pela inexistência de políticas de adaptação adequadas na região de origem (OJIMA, 2015), são determinantes que, de forma conjunta aos estressores ambientais, operam sobre as decisões de migração dos indivíduos (BARBIERI, 2011).

No que concerne aos fatores que condicionam a mobilidade, Hugo (1996) defende que as decisões de migrar ou permanecer são tomadas pelo indivíduo ao longo de um continuum onde de um lado estariam as motivações puramente econômicas, melhor definidas pela mobilidade voluntária, e no sentido oposto se posicionariam os movimentos forçados, característicos dos migrantes ambientais. A partir dessa leitura, Barbieri (2011) conclui que "perfis puros" são raros e constituem casos difíceis de observar empiricamente. Outro detalhe importante é que os migrantes podem ser em muitos dos casos mais vulneráveis do que os que lá já residem há algum tempo, embora o estabelecimento de redes e capital social possa reduzir o grau de exposição desses indivíduos ao risco e suas vulnerabilidades (ADGER et al., 2015; BLACK et al., 2011).

Nesse sentido, é necessário construir um diálogo mais amplo que incorpore tanto a vulnerabilidade à seca e as respostas adaptativas, considerando a multiplicidade das escalas, quanto as implicações para as regiões receptoras de migrantes. Assim, tanto a mobilidade em seu sentido mais amplo quanto a urbanização nos municípios do semiárido podem, em que pesem algumas consequências negativas, serem alternativas para reduzir a vulnerabilidade (OJIMA, 2015; VANWEY; GUEDES; D'ANTONA, 2012).

Ademais, a seca, além de outros agentes como político-econômicos e sociais, como os programas sociais de um modo geral e as estratégias de sobrevivência e adaptação à seca, devem ser concebidos como problemas interconectados (RIBOT, 1996; SIVAKUMAR; DAS; BRUNINI, 2005), como contemplados neste trabalho.

\section{MATERIAIS E MÉTODOS}

A principal fonte de dados utilizada neste estudo é um survey realizado com 1.064 domicílios urbanos do Seridó potiguar. O Seridó que tratamos aqui corresponde a duas microrregiões do estado do Rio Grande do Norte integralmente incluídas no semiárido brasileiro. Essa região possui 17 municípios, a saber: Caicó, Ipueira, Jardim de Piranhas, São Fernando, São João do Sabugi, Serra Negra do Norte, Timbaúba dos Batistas, Acari, Carnaúba dos Dantas, Cruzeta, Currais Novos, Equador, Jardim do Seridó, Ouro Branco, Parelhas, Santana do Seridó e São José do Seridó.

Trata-se, ao nosso ver, da primeira pesquisa amostral do gênero, com representatividade amostral na escala microrregional e com um conjunto amplo de informações que permitem associar fatores socioeconômicos, demográficos e institucionais a informações objetivas e subjetivas sobre secas e qualidade ambiental. A pesquisa de campo foi realizada em cerca de três semanas e teve uma duração média de 35 minutos cada entrevista.

A estratégia foi a utilização de uma amostra probabilística, com probabilidades de seleção em três estágios (município, setor censitário e domicílio). A amostra é representativa da população urbana do Seridó potiguar. No primeiro estágio foi adotada a metodologia de amostragem aleatória proporcional ao número de setores urbanos para selecionar os municípios. No segundo, no qual os setores foram selecionados, a amostragem foi do tipo aleatória simples. Porém, os setores selecionados foram fixados em $20 \%$ dos setores urbanos de cada município. No terceiro estágio, a metodologia de amostragem foi a aleatória estratificada.

Os estratos tiveram como critério a renda domiciliar per capita média dos domicílios nos setores urbanos, definidos como: 1) domicílios com renda per capita média inferior ou igual a $\mathrm{R} \$ 300,00$ mensais para as pessoas de 10 anos e mais (estrato 1) e 2) domicílios com renda per capita média superior a $\mathrm{R} \$ 300,00$ mensais para as pessoas de 10 anos e mais.

O trabalho contou com a participação de seis bolsistas de Desenvolvimento Tecnológico e Industrial (DTI-C) do CNPq, mais dois estudantes de pós-graduação voluntários, sendo um deles coautor deste trabalho. Além do apoio do CNPq, o survey contou com o financiamento da Rede Brasileira de Pesquisas sobre Mudanças Climáticas Globais (Rede Clima). 
Para analisar as estratégias de adaptação aos meios de subsistência, trabalhamos com estatísticas descritivas de grupos populacionais específicos relacionados à condição de mobilidade dos indivíduos. As análises conduzidas neste trabalho dizem respeito apenas aos indivíduos que residiam em domicílios urbanos em 2017 e que responderam às perguntas de percepção sobre as secas. Em todos os domicílios da nossa amostra uma pessoa respondeu a essas perguntas, correspondendo a uma amostra de 1.064 indivíduos. Ao expandir a amostra, esses indivíduos representaram 59.268 pessoas na população da região do Seridó.

Os indivíduos, por sua vez, foram distribuídos nas seguintes categorias:

- Indivíduo que pratica mobilidade cotidiana ou sazonal - indivíduo que, na condição de residente do Seridó no período da pesquisa, respondeu que ia a outro município com frequência;

- Indivíduo que residia em outro município - indivíduo que, na condição de residente do Seridó no período da pesquisa, respondeu que já residiu em outro município;

- Indivíduo que residia em outro município e praticava mobilidade cotidiana ou sazonal indivíduo que, na condição de residente do Seridó no período da pesquisa, respondeu que já residiu em outro município e que simultaneamente ia a outro município com frequência;

- Indivíduo imóvel - indivíduo que, na condição de residente do Seridó no período da pesquisa, afirmou que não pratica nenhum dos tipos de mobilidade anteriores.

Adicionalmente, comparamos os indivíduos segundo as tipologias de mobilidade definidas e as seguintes variáveis: condição de beneficiário de programas sociais, Razão de Sexo², ramo de atividade da ocupação e perguntas sobre os motivos que levariam a migrar, se é mais difícil viver na zona urbana diante da seca, e se esses indivíduos já pensaram em migrar por causa da seca.

Essas variáveis foram escolhidas porque elas refletem em estratégias demográficas e/ou econômicas para lidar com as situações de risco e aproveitar oportunidades econômicas. Neste artigo, buscamos entender como as dimensões sociodemográficas, por meio da mobilidade, estrutura etária, sexo, percepção sobre a seca e ocupação refletem em respostas de adaptação aos meios de subsistência no semiárido (BILSBORROW, 1987; STARK; BLOOM, 1985).

Assim, determinadas situações de risco podem representar uma oportunidade para estabelecimento de relações diretas e indiretas com mercados urbanos (SHERBININ et al., 2008), como a dupla residência que permite por exemplo os acessos a empregos urbanos e serviços básicos, crédito e, portanto, se convertem em respostas adaptativas. Ao mesmo tempo, a mobilidade pode representar um risco na medida em que as vulnerabilidades específicas acompanham os migrantes ou podem ser uma forma de adaptação (BARBIERI et al., 2010), o que depende muito da capacidade das instituições (ADGER et al., 2015; BLACK et al., 2011), bem como das políticas sociais, o que torna essa análise necessária.

\section{VULNERABILIDADE E MOBILIDADE NO SEMIÁRIDO BRASILEIRO}

O semiárido brasileiro engloba 1.133 municípios e cerca de 22,5 milhões de habitantes, dos quais mais de 21 milhões residem no Nordeste. Essa região é uma das semiáridas mais povoadas do mundo (AB'SABER, 1999). Em se tratando do Nordeste, praticamente todos os estados estão incluídos no semiárido, com exceção do Maranhão. Em contrapartida, Minas Gerais tem 85 municípios que estão na área semiárida delimitada pelo Ministério da Integração Nacional (MIN) em 2005 (BRASIL, 2005; OJIMA, 2015).

Se o Brasil possuía 5.565 municípios em 2010, isso significa dizer que mais de 1/5 desses municípios são atingidos pelo fenômeno da seca (BRASIL, 2005). Embora os deslocamentos populacionais em massa com destino à Região Sudeste ao longo dos 50 anos fossem facilmente associados a fatores de atração migratória para centros urbanos mais dinâmicos, não podemos ignorar o fenômeno natural das secas que atinge $11,8 \%$ da população do País (OJIMA, 2015). 
No entanto, a seca não atinge somente a região semiárida do Nordeste, e muito menos as outras áreas da região estarão imunes dos efeitos adversos das mudanças climáticas. Para a Zona da Mata, mata extensa localizada na zona costeira onde mantém desde a época do Brasil-Colônia plantações de cana-de-açúcar e de cacau, as secas extremas também são ameaças (DE NYS; ENGLE; MAGALHÃES, 2016), embora o aumento da temperatura média e as inundações sejam questões mais preocupantes para a região (IPCC, 2012, 2013).

O Agreste, por outro lado, é uma área de transição do semiárido para a Zona da Mata, tanto que está incluído no polígono das secas. Nessa área, a produção de gêneros alimentícios e a pecuária de pequena escala, bem como a população que reside nesse bioma, também são afetadas pelas estiagens (DE NYS; ENGLE; MAGALHÃES, 2016).

Em relação à mobilidade como resposta aos períodos de seca, principalmente até a década de 1970, a população buscava melhores condições de vida na região mais dinâmica do País, o Sudeste, trazendo consigo vários problemas para os centros urbanos, inclusive suas vulnerabilidades específicas - muito embora os fluxos migratórios tenham sido, pelo menos de forma majoritária, analisados sob a ótica dos fatores de atração da região de destino. A rigor, diversos estudos têm tratado da precariedade da inserção e consequente (re)criação de situações de vulnerabilidade dos imigrantes nordestinos, particularmente nas grandes metrópoles a problemática da inserção pode ser exemplificada em diversos estudos (DENALDI et al., 2016; LOBO et al., 2015; SILVA; TRAVASSOS, 2009).

Mesmo em períodos mais recentes, mais de $75 \%$ dos municípios de toda a região que compreende os estados do Piauí, Ceará, Rio Grande do Norte, Paraíba, Pernambuco, Alagoas, Sergipe, Bahia e Minas Gerais possuem, mesmo que em menores níveis se comparado às décadas anteriores, Taxas Líquidas de Migração (TLM) negativas, conforme se observa na Figura 2. Isso significa que a maior parte do semiárido tem características de expulsão de população que, notadamente, se associa às desigualdades regionais. Por outro lado, a partir da Figura 2 é possível perceber que existem particularidades dentro desses estados no que diz respeito à dinâmica migratória da região. As TLM negativas mais altas concentram-se em sua maior parte na porção semiárida dos estados, diferente do que acontece com os municípios do entorno. Em particular, os municípios do norte de Minas Gerais e os municípios costeiros dos estados apresentam taxas positivas e que os diferenciam da porção semiárida.

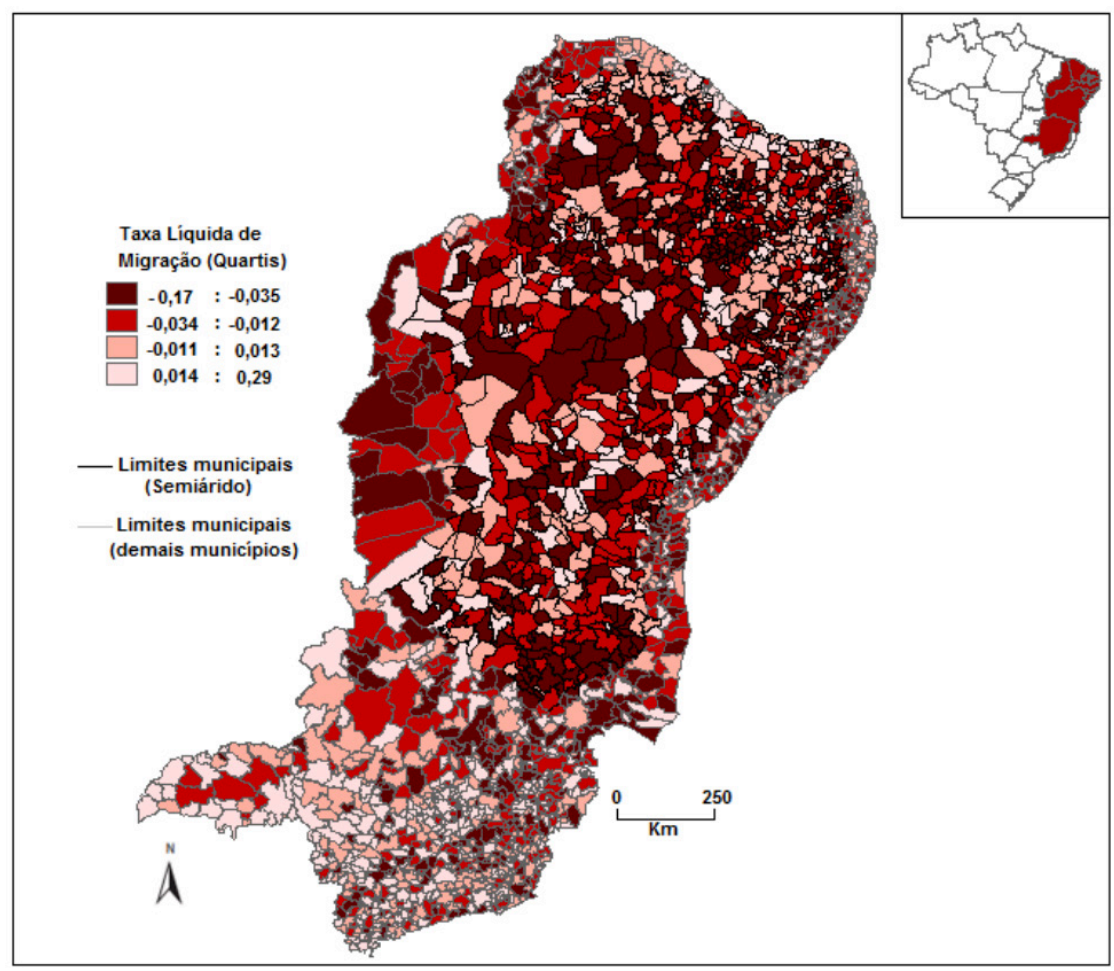

Figura 2 | Semiárido brasileiro: Taxa Líquida de Migração (TLM), municípios do semiárido e municípios do entorno, $2005 / 2010$ 
O que parece contraditório é que apesar de a Figura 2 mostrar que os municípios incluídos no semiárido possuem TLM maiores em relação aos demais, a maioria dos indivíduos entrevistados no Seridó potiguar respondeu que nunca pensou em mudar de cidade por causa da seca, conforme se observa na Tabela 1. Parece pouco consistente afirmar que a seca não tenha provocado efeito sobre os fluxos migratórios na região, muito embora estudos semelhantes tenham apontado que os períodos de seca aumentaram a probabilidade de migração por motivo de trabalho em algumas regiões (GRAY; MUELLER, 2012) e que os indivíduos podem adotar outras estratégias de adaptação além da migração (BARDSLEY; HUGO, 2010).

Tabela 1 | Seridó potiguar: tipologias de mobilidade dos indivíduos que vivem em domicílios com ou sem emigrante segundo a percepção sobre migração e a vida na zona urbana diante da seca, 2017.

\begin{tabular}{|c|c|c|c|c|c|c|c|c|}
\hline $\begin{array}{l}\text { Individuos vivendo em domicílios com } \\
\text { emigrante }\end{array}$ & \multicolumn{4}{|c|}{$\begin{array}{c}\text { Diante da seca é mais fácil viver na } \\
\text { zona urbana? }\end{array}$} & \multicolumn{4}{|c|}{$\begin{array}{l}\text { Já pensou em se mudar por causa do } \\
\text { seca? }\end{array}$} \\
\hline TIPOLOGIAS DE MOBILIDADE & SIM & $\%$ & NÃO & $\%$ & SIM & $\%$ & NÃO & $\%$ \\
\hline (1) INDIVÍDUOS IMÓVEIS & 2.467 & 94,1 & 154 & 5,9 & 674 & 25,7 & 1.947 & 74,3 \\
\hline $\begin{array}{l}\text { (2) INDIVÍDUOS QUE PRATICAM } \\
\text { MOBILIDADE COTIDIANA OU } \\
\text { SAZONAL }\end{array}$ & 1.577 & 91,5 & 147 & 8,5 & 585 & 33,9 & 1.140 & 66,1 \\
\hline $\begin{array}{l}\text { (3) INDIVÍDUOS QUE RESIDIRAM EM } \\
\text { OUTRO MUNICÍPIO }\end{array}$ & 3.579 & 84,7 & 646 & 15,3 & 1.015 & 24,0 & 3.210 & 76,0 \\
\hline $\begin{array}{l}\text { (4) INDIVÍDUOS QUE RESIDIRAM } \\
\text { EM OUTRO MUNICÍPIO E PRATICAM } \\
\text { MOBILIDADE COTIDIANA OU } \\
\text { SAZONAL }\end{array}$ & 1.078 & 84,8 & 193 & 15,2 & 305 & 24,0 & 966 & 76,0 \\
\hline TOTAL & 8.701 & 88,4 & 1.140 & 11,6 & 2.579 & 7,9 & 30.171 & 92,1 \\
\hline $\begin{array}{l}\text { Indivíduos vivendo em domicílios sem } \\
\text { emigrante }\end{array}$ & \multicolumn{4}{|c|}{$\begin{array}{c}\text { Diante da seca é mais fácil viver na } \\
\text { zona urbana? }\end{array}$} & \multicolumn{4}{|c|}{$\begin{array}{l}\text { Já pensou em se mudar por causa da } \\
\text { seca? }\end{array}$} \\
\hline TIPOLOGIAS DE MOBILIDADE & SIM & $\%$ & NÃO & $\%$ & SIM & $\%$ & NÃO & $\%$ \\
\hline (1) INDIVÍDUOS IMÓVEIS & 10.998 & 86,6 & 1.701 & 13,4 & 2.460 & 19,2 & 10.321 & 80,8 \\
\hline $\begin{array}{c}\text { (2) INDIVÍDUOS QUE PRATICAM } \\
\text { MOBILIDADE COTIDIANA OU } \\
\text { SAZONAL }\end{array}$ & 6.073 & 77,7 & 1.742 & 22,3 & 1.731 & 22,3 & 6.047 & 77,7 \\
\hline $\begin{array}{l}\text { (3) INDIVÍDUOS QUE RESIDIRAM EM } \\
\text { OUTRO MUNICÍPIO }\end{array}$ & 14.758 & 79,4 & 3.838 & 20,6 & 5.030 & 26,6 & 13.889 & 73,4 \\
\hline $\begin{array}{l}\text { (4) INDIVÍDUOS QUE RESIDIRAM } \\
\text { EM OUTRO MUNICÍPIO E PRATICAM } \\
\text { MOBILIDADE COTIDIANA OU } \\
\text { SAZONAL }\end{array}$ & 7.929 & 81,3 & 1.829 & 18,7 & 2.056 & 20,9 & 7.801 & 79,1 \\
\hline TOTAL & 39.758 & 81,4 & 9.110 & 18,6 & 11.277 & 22,9 & 38.058 & 77,1 \\
\hline
\end{tabular}

Fonte: Survey Seridó Potiguar, 2017.

Por outro lado, as secas também podem incentivar respostas adaptativas de curto prazo tais como outros tipos de mobilidade. A partir da Tabela 1 observamos que em todas as categorias de mobilidade apresentadas, os indivíduos responderam majoritariamente que nunca pensaram em mudar de cidade por causa da seca, independente da presença de emigrantes no domicílio. Não aparenta existir, além disso, diferenças consideráveis nesse quesito quando em se tratando dos quatro tipos de mobilidade analisadas.

Quando perguntamos se é mais fácil viver na zona urbana diante da seca, porém, houve uma inversão nas respostas com maiores proporções para aqueles que responderam de forma afirmativa. Especialmente para os indivíduos que não fazem nenhum tipo de mobilidade, a proporção dos que responderam que 
é mais fácil viver na zona urbana diante da seca foi bastante elevada $(94,1 \%)$, conforme se observa na Tabela 1.

Não há também, nesse caso, grandes diferenças nos níveis das respostas quando observamos as categorias 3 e 4 de mobilidade. Porém, há indicação de uma diferença importante nas respostas quando consideramos a categoria 2 (Indivíduos que praticam mobilidade cotidiana ou sazonal) - cerca de $14 \%$ maior para domicílios com emigrantes - e secundariamente para a categoria 1 (Indivíduos imóveis) cerca de $8 \%$ maior para domicílios com emigrantes. Há evidência, nesse caso, de que a presença de emigrantes prévios pode favorecer uma percepção mais positiva da vida urbana como adaptação à seca em domicílios com menor mobilidade em termos de alcance espacial e que não implica mudança permanente de residência (imobilidade ou mobilidade temporária).

Um detalhe interessante é que esses indivíduos já vivem na zona urbana, o que pode sinalizar uma decisão tomada estrategicamente para se adaptar aos períodos de estiagem. Embora essa afirmação seja pouco consistente dado que não sabemos exatamente se esses indivíduos residiram na zona rural anteriormente, isso nos permite uma reflexão sobre a escala de atuação da seca, sobretudo a escala social e as respostas adaptativas resultantes. Nesse sentido, se faz relevante compreender a atuação e a interação entre esses fenômenos (mobilidade e mudanças ambientais) para além dos limites políticoadministrativos.

A explicação para esses resultados está, a nosso ver, na própria multidimensionalidade das causas da mobilidade. Por exemplo, a relação dos fenômenos ambientais com eventos demográficos, sobretudo a mobilidade, é bastante complexa. $\mathrm{E}$, especialmente em se tratando da relação entre a mobilidade e a seca, conhecemos ainda muito pouco, apesar de nossa experiência histórica. A rigor, essa relação pode ser alterada dependendo de contextos institucionais específicos e da escala de atuação desses fenômenos, o que nem sempre é conhecido (GIBSON; OSTROM; AHN, 2000). Qual a escala espacial de atuação da seca? Existe uma escala social de atuação dos fenômenos ambientais? Provavelmente precisamos ser cautelosos com as respostas, se é que existe uma resposta completa, especialmente porque são relações bastante complexas de se examinar. No entanto, as políticas de mitigação e adaptação devem garantir que processos de desigualdades já existentes não sejam sobrepostos (DENTON, 2002), devido ao que chamamos de distribuição desigual de capacidades adaptativas (HANDMER; DOVERS; DOWNING, 1999).

A Tabela 2 mostra que, apesar da maior parte dos indivíduos ter afirmado que não mudaria de cidade por causa da seca, a migração por motivo de trabalho (cerca de $31 \%$ do total de respostas) sinaliza uma resposta endógena em que a seca seria uma forma de afetar os meios de subsistência e tal processo resultaria no deslocamento populacional. A migração, muito embora também possa ser adiada até que todas as alternativas in situ sejam esgotadas (NAWROTZKI; DEWAARD, 2016).

Especialmente no caso das secas, que possibilitam respostas diferenciadas (GRAY; MUELLER, 2012), à medida que os seus efeitos sobre os meios de sobrevivência dos indivíduos são de início lento, permitem que estes tenham tempo para fornecer respostas de curto prazo. Ainda assim, a Tabela 2 mostra que a seca continua sendo mencionada, inclusive por indivíduos que já residiram em outro município, quando perguntados sobre o que os levariam a mudar de cidade.

Outro aspecto importante é como as tipologias de mobilidade se comportam em relação aos motivos que levariam a migrar. Uma das principais observações que fazemos a partir dos resultados da Tabela 2 é que existe certo equilíbrio na proporção das respostas por motivo de trabalho, exceto para os indivíduos que residiram em outro município e ao mesmo tempo praticam mobilidade cotidiana ou sazonal. As maiores proporções de indivíduos que tiveram como resposta a seca, por sua vez, foram para os indivíduos menos móveis, com exceção para o caso dos indivíduos que praticam mobilidade pendular (12\%). 
Tabela 2 | Seridó Potiguar: tipologias de mobilidade dos indivíduos segundo os motivos que os levariam a migrar, 2017.

\begin{tabular}{|c|c|c|c|c|c|c|c|c|c|c|}
\hline \multirow[t]{2}{*}{$\begin{array}{c}\text { Tipologias de } \\
\text { mobilidade }\end{array}$} & \multicolumn{10}{|c|}{ Que motivo o levaria a mudar de cidade? } \\
\hline & SECA & $\%$ & TRABALHO & $\%$ & FAMÍLIA & $\%$ & OUTROS & $\%$ & NENHUM & $\%$ \\
\hline $\begin{array}{l}\text { (1) } \\
\text { INDIVÍDUOS } \\
\text { IMÓVEIS }\end{array}$ & 3.431 & 22,7 & 4.999 & 33,1 & 1.056 & 7,0 & 3.100 & 20,5 & 2.527 & 16,7 \\
\hline $\begin{array}{c}(2) \\
\text { INDIVÍDUOS } \\
\text { QUE } \\
\text { PRATICAM } \\
\text { MOBILIDADE } \\
\text { COTIDIANA } \\
\text { OU SAZONAL }\end{array}$ & 1.138 & 12,0 & 2.737 & 28,8 & 913 & 9,6 & 3.975 & 41,8 & 745 & 7,8 \\
\hline $\begin{array}{c}\text { (3) } \\
\text { INDIVÍDUOS } \\
\text { QUE } \\
\text { RESIDIRAM } \\
\text { EM OUTRO } \\
\text { MUNICÍPIO }\end{array}$ & 4.117 & 18,3 & 7.292 & 32,5 & 1.783 & 7,9 & 4.110 & 18,3 & 5.163 & 23,0 \\
\hline $\begin{array}{c}\text { (4) } \\
\text { INDIVÍDUOS } \\
\text { QUE } \\
\text { RESIDIRAM } \\
\text { EM OUTRO } \\
\text { MUNICÍPIO } \\
\text { E PRATICAM } \\
\text { MOBILIDADE } \\
\text { COTIDIANA } \\
\text { OU SAZONAL }\end{array}$ & 1.812 & 16,8 & 2.756 & 25,5 & 1.243 & 11,5 & 3.477 & 32,2 & 1.524 & 14,1 \\
\hline TOTAL & 10.498 & 18,1 & 17.784 & 30,7 & 4.995 & 8,6 & 14.662 & 25,3 & 9.959 & 17,2 \\
\hline
\end{tabular}

Fonte: Survey Seridó Potiguar, 2017.

A mobilidade, apesar de poder ser uma forma de gerar novos riscos e vulnerabilidades, também pode estar fortemente relacionada à capacidade de resposta de grupos populacionais específicos. Nesse sentido, políticas públicas que promovam a mobilidade de forma segura, além de possibilitarem o engajamento desses indivíduos por meio da integração de atividades agrícolas e não agrícolas (SHERBININ et al., 2008; VANWEY; GUEDES; D'ANTONA, 2012) e da busca por serviços públicos (OJIMA, 2015), podem ser entendidas como uma ferramenta para alcançar a justiça socioambiental por meio da distribuição social dos riscos (ADGER et al., 2015; BLACK et al., 2011).

No caso particular da seca no Nordeste brasileiro, ao observar a nossa experiência histórica com perda populacional para outras regiões, a vulnerabilidade das instituições e as medidas da "Política da Seca" que foram iniciadas há quase um século (BURSZTYN, 2003, p. 76) pouco nos leva a crer que medidas de adaptação in situ efetivas puderam ser concretizadas.

A adaptação às secas no semiárido é, acima de tudo, uma questão social na medida em que situações específicas de vulnerabilidade são entendidas como uma complexa intersecção de fatores, como pobreza, ecossistemas em crise e a falta ou ineficiência de intervenções locais (ACNUR, 2012; MORTON; BONCOUR; LACZKO, 2008). Desse modo, pesquisas recentes têm mostrado que os pobres rurais e dependentes de atividades agropastoris são mais vulneráveis às mudanças ambientais. Além disso, a capacidade de resposta via mobilidade é mais limitada devido aos custos iniciais que são impostos (CORREIA; OJIMA, 2017; NAWROTZKI; DEWAARD, 2016).

Apesar de haver certa homogeneidade em relação às motivações para migrar, principalmente naquelas relacionadas à seca, os indivíduos imóveis são mais dependentes de atividades agrícolas como podemos ver na Tabela 3. Em se tratando dos domicílios nos quais pelo menos um membro mora fora 
do município, praticamente não existe inserção em atividade agrícola. Desse modo, a mobilidade pode revelar uma estratégia relevante dos domicílios para acessar capitais (SHERBININ et al., 2008), muito embora não pareça uma oportunidade acessível a todos os grupos populacionais.

Entre as categorias de mobilidade apresentadas, a Tabela 3 mostra que praticamente não existem diferenças nas proporções, com uma maior participação feminina em ambas as categorias. No entanto, para os indivíduos imóveis, a proporção de mulheres também é superior. O que chama bastante atenção, por outro lado, é que a proporção de mulheres imóveis que vivem em domicílios sem emigrante $(81,5 \%)$ é superior em relação aos domicílios com emigrante $(65,6 \%)$, sinalizando que existem diferenciais importantes nesses domicílios, o que pode subsidiar oportunidades de análises exploratórias muito férteis para discutir a relação entre mobilidade e estratégias de adaptação às secas.

Tabela 3 | Seridó Potiguar: tipologias de mobilidade dos indivíduos que vivem em domicílios com ou sem emigrante segundo o sexo e a ocupação, 2017.

\begin{tabular}{|c|c|c|c|c|c|c|c|c|}
\hline $\begin{array}{l}\text { Indivíduos vivendo em domicílios com } \\
\text { emigrante }\end{array}$ & \multicolumn{4}{|c|}{ Sexo } & \multicolumn{4}{|c|}{ Ocupado em atividade agrícola? } \\
\hline TIPOLOGIAS DE MOBILIDADE & MASCULINO & $\%$ & FEMININO & $\%$ & SIM & $\%$ & NÃO & $\%$ \\
\hline (1) INDIVÍDUOS IMÓVEIS & 901 & 34,4 & 1.720 & 65,6 & 0 & 0,0 & 2.621 & 100,0 \\
\hline $\begin{array}{l}\text { (2) INDIVÍDUOS QUE PRATICAM } \\
\text { MOBILIDADE COTIDIANA OU } \\
\text { SAZONAL }\end{array}$ & 515 & 29,9 & 1.210 & 70,1 & 0 & 0,0 & 1.725 & 100,0 \\
\hline $\begin{array}{l}\text { (3) INDIVÍDUOS QUE RESIDIRAM } \\
\text { EM OUTRO MUNICÍPIO }\end{array}$ & 778 & 18,4 & 3.447 & 81,6 & 390 & 9,2 & 3.835 & 90,8 \\
\hline $\begin{array}{l}\text { (4) INDIVÍDUOS QUE RESIDIRAM } \\
\text { EM OUTRO MUNICÍPIO E } \\
\text { PRATICAM MOBILIDADE } \\
\text { COTIDIANA OU SAZONAL }\end{array}$ & 493 & 38,8 & 778 & 61,2 & 77 & 6,1 & 1.194 & 93,9 \\
\hline TOTAL & 2.687 & 27,3 & 7.155 & 72,7 & 467 & 4,7 & 9.375 & 95,3 \\
\hline $\begin{array}{l}\text { Indivíduos vivendo em domicílios sem } \\
\text { emigrante }\end{array}$ & \multicolumn{4}{|c|}{ Sexo } & \multicolumn{4}{|c|}{ Ocupado em atividade agrícola } \\
\hline TIPOLOGIAS DE MOBILIDADE & MASCULINO & $\%$ & FEMININO & $\%$ & SIM & $\%$ & NÃO & $\%$ \\
\hline (1) INDIVÍDUOS IMÓVEIS & 2.367 & 18,5 & 10.414 & 81,5 & 275 & 2,2 & 12.506 & 97,8 \\
\hline $\begin{array}{l}\text { (2) INDIVÍDUOS QUE PRATICAM } \\
\text { MOBILIDADE COTIDIANA OU } \\
\text { SAZONAL }\end{array}$ & 2.151 & 27,4 & 5.694 & 72,6 & 924 & 11,8 & 6.922 & 88,2 \\
\hline $\begin{array}{c}\text { (3) INDIVÍDUOS QUE RESIDIRAM } \\
\text { EM OUTRO MUNICÍPIO }\end{array}$ & 3.809 & 20,1 & 15.110 & 79,9 & 709 & 3,7 & 18.210 & 96,3 \\
\hline $\begin{array}{l}\text { (4) INDIVÍDUOS QUE RESIDIRAM } \\
\text { EM OUTRO MUNICÍPIO E } \\
\text { PRATICAM MOBILIDADE } \\
\text { COTIDIANA OU SAZONAL }\end{array}$ & 2.690 & 27,2 & 7.191 & 72,8 & 514 & 5,2 & 9.367 & 94,8 \\
\hline TOTAL & 11.017 & 22,3 & 38.409 & 77,7 & 2.422 & 4,9 & 47.005 & 95,1 \\
\hline
\end{tabular}

Fonte: Survey Seridó Potiguar, 2017.

Ainda assim, cabe aqui sinalizar que a composição desses domicílios sintetiza algumas estratégias de adaptação como foi constatado em pesquisas recentes para a região (CORREIA; OJIMA, 2018).

Muito embora nossos dados nos limitem na medida em que desconhecemos os indivíduos que emigraram em relação à idade e ao sexo, por exemplo, essas estratégias podem sinalizar processos de exclusão não só em relação a grupos populacionais com níveis de renda distintos, mas também em relação a questões de gênero muito fortes, que poderão ser reafirmadas caso comprovada uma maior emigração masculina originária dessa região. Uma conexão que convém estabelecer nesse caso é que, sendo as mulheres usufruidoras e representantes dos domicílios diante dos programas sociais do governo, esses programas sociais, além de possibilitar respostas de curto prazo via mobilidade, ajudam a equalizar essas relações de gênero e o poder de barganha sobre as decisões de mobilidade desses domicílios. 


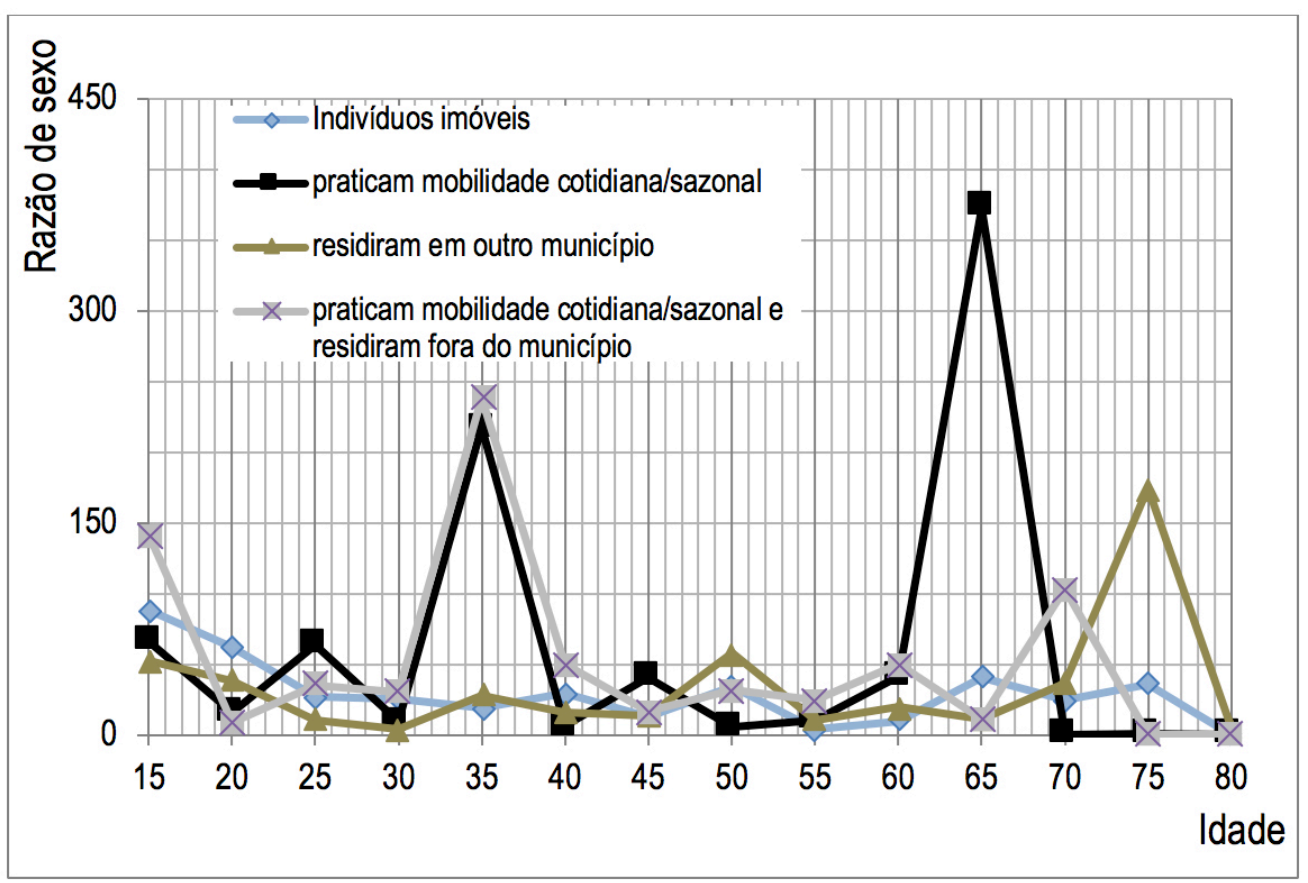

Figura 3 | Seridó Potiguar: Razão de Sexo por tipologias de mobilidade praticadas pelos indivíduos, 2017.

Fonte: Survey Seridó Potiguar, 2017.

Corroborando esse raciocínio, a Figura 3 mostra um protagonismo feminino em relação às estratégias de mobilidade no Seridó potiguar. Com poucas exceções, a Razão de Sexo é inferior a 100, indicando uma maior participação feminina em ambos os tipos de mobilidade.

Nesse caso, uma Razão de Sexo igual a 100 indicaria paridade entre homens e mulheres. Exceto para alguns grupos de idade dos indivíduos que praticam mobilidade cotidiana ou sazonal (35-39 e 65-69 anos), os que já residiram em outro município (75-79 anos) e para os que estão incluídos em mais de um dos tipos de mobilidade analisados (15-19, 35-39 e 75-79 anos), a Razão de Sexo é superior a 100, indicando uma maior participação masculina nesses grupos. Por outro lado, como apontam os estudos clássicos de migração (LEE, 1966; RAVENSTEIN, 1889), esse protagonismo feminino é conjugado a uma resposta usualmente de curto prazo ao fenômeno da seca, com maior expressividade para os movimentos de curtas distâncias.

Assumindo que a migração é uma alternativa de adaptação para aqueles que possuem recursos suficientes para se deslocarem, a população mais empobrecida terá mais dificuldades de adaptar-se às secas in situ ou ex situ. Uma maior mobilidade tem suas consequências negativas e desafios para as instituições, mas, por outro lado, pode representar um mecanismo de geração de novos riscos e vulnerabilidades. Complementarmente, os migrantes que saem do semiárido em direção às grandes metrópoles podem ser em muitos dos casos mais vulneráveis do que os que permanecem na região de origem ou os que já residem nas regiões de destino dos migrantes. Nesse aspecto, o estabelecimento de redes e capital social pode reduzir o grau de exposição desses indivíduos ao risco e suas vulnerabilidades (ADGER et al., 2015; BLACK et al., 2011).

Essa preocupação em entender os impactos dos migrantes para as regiões de destino tem sido recorrente, especialmente no que concerne ao planejamento urbano nas regiões receptoras desses migrantes em detrimento das situações de vulnerabilidade nas regiões de expulsão. Isso justifica-se pelo fato de as áreas urbanas concentrarem uma parcela da população brasileira que está em torno da metade e tal necessidade de estudos e políticas públicas específicas. Entretanto, os problemas ambientais urbanos são muito mais complexos e é necessário admitir que eles assumem singularidades diante de contextos regionais distintos (IBGE, 2010; OJIMA, 2015). 
Outra questão importante diz respeito ao ritmo de crescimento e de urbanização dos municípios, ambos cada vez mais intensos no semiárido brasileiro. Quanto a isso, destacamos que a partir da década de 1980 tem ocorrido uma mudança relevante nos fluxos migratórios que traz consigo uma nova configuração no que diz respeito à distribuição espacial da população. A atração das grandes cidades do Sudeste parece não ter mais o mesmo peso, e regiões como o Nordeste, que outrora perdiam população para outras localidades, recebem de volta um contingente considerável de pessoas com o incremento da migração de retorno (CUNHA, 2005).

Seguindo essa tendência, a maior parte dos migrantes intermunicipais de retorno ao semiárido (72,5\%) fixou residência em áreas urbanas dentro da própria região. Apesar do seu histórico como uma região emissora de migrantes, principalmente no sentido rural-urbano, essa característica tem se modificado, visto que pessoas que deixaram o semiárido em décadas passadas retornaram recentemente para os seus municípios, porém, escolheram residir em áreas urbanas.

Em se tratando do Nordeste de um modo geral, os que integram o semiárido já são menos urbanos que os demais. Os municípios da região que não integram o semiárido, por outro lado, concentram mais de $70 \%$ da população em áreas urbanas, enquanto em alguns municípios, como os do semiárido piauiense, por exemplo, possuem taxas de urbanização muito inferiores com metade da população residindo na zona rural. Uma tendência global é que as pessoas se concentrem cada vez mais nas áreas urbanas, trazendo desafios para o planejamento urbano. No semiárido, esse processo pode ser acentuado com a migração de retorno, conforme se defende no parágrafo anterior.

Embora no imaginário social a seca seja um fenômeno ambiental que predomina em toda a região nordestina, nela convivem duas características bem distintas: enquanto os períodos de estiagem, tradicionalmente associados ao cenário regional como um todo, dominam a porção semiárida dos estados, os desastres naturais atingem as áreas litorâneas com altos índices de ocorrências de chuvas (OJIMA, 2015).

Devido às mudanças climáticas, a tendência é que esses eventos se intensifiquem e elevem o nível do mar. E quanto a essa questão, é importante também reforçar que as cidades litorâneas abrigam cerca de $18 \%$ da população urbana brasileira (OJIMA; MARTINE, 2012). Assim, principalmente as regiões metropolitanas, que compreendem uma porção expressiva das áreas costeiras, representam um potencial risco tanto para a população que nela já residia quanto para os que outrora buscam refúgio das secas.

A urbanização dos municípios do semiárido poderia também ser uma alternativa para reduzir substanciais impactos ambientais causados pela exploração dos recursos naturais nas áreas rurais (OJIMA, 2015), além de amenizar os problemas decorrentes da alta densidade e expansão de áreas de risco nos grandes centros metropolitanos. Entretanto, o processo histórico e estudos para outras regiões sinalizam que o ritmo acelerado de crescimento desses municípios apresenta desafios importantes para o planejamento urbano, pois as formas de uso e ocupação do solo podem acentuar processos de exclusão social em áreas urbanas e de degradação ambiental em áreas rurais, devido ao aumento da demanda por serviços ecossistêmicos (SHERBENIN et al., 2008; VANWEY; GUEDES; D'ANTONA, 2012).

Desse modo, as secas, mais do que nunca, representam um desafio para os governos e instituições. Embora os fluxos de longa distância, especialmente do Nordeste para outras regiões, a partir da década de 1980 tenham perdido sua força (CUNHA, 2005), a mobilidade em menores escalas de tempo e espaço como estratégia de adaptação às secas e às mudanças climáticas tem figurado uma ferramenta de análise mais eficaz dessa problemática. Nesse aspecto, os programas sociais apresentam um significado relevante para a região. A Tabela 4 mostra que nos domicílios com emigrante a proporção de indivíduos que recebem algum dos benefícios sociais do governo (31,5\%) é superior ao mesmo percentual para indivíduos que vivem em domicílios sem emigrante $(29,9 \%)$, embora com pouca diferença. 
Tabela 4 | Seridó Potiguar: tipologias de mobilidade praticadas pelos indivíduos que vivem em domicílios com ou sem emigrante e a condição de beneficiário de programa de transferência de renda, 2017.

\begin{tabular}{|c|c|c|c|c|}
\hline $\begin{array}{c}\text { Indivíduos vivendo em domicílios com } \\
\text { emigrante }\end{array}$ & \multicolumn{4}{|c|}{ Recebe benefício social? } \\
\hline TIPOLOGIAS DE MOBILIDADE & SIM & $\%$ & NÃO & $\%$ \\
\hline (1) INDIVÍDUOS IMÓVEIS & 988 & 39,6 & 1.509 & 60,4 \\
\hline $\begin{array}{l}\text { (2) INDIVÍDUOS QUE PRATICAM } \\
\text { MOBILIDADE COTIDIANA OU SAZONAL }\end{array}$ & 85 & 5,4 & 1.483 & 94,6 \\
\hline $\begin{array}{c}\text { (3) INDIVÍDUOS QUE RESIDIRAM EM } \\
\text { OUTRO MUNICIIPIO }\end{array}$ & 1.427 & 38,2 & 2.304 & 61,8 \\
\hline $\begin{array}{l}\text { (4) INDIVÍDUOS QUE RESIDIRAM } \\
\text { EM OUTRO MUNICÍPIO E PRATICAM } \\
\text { MOBILIDADE COTIDIANA OU SAZONAL }\end{array}$ & 145 & 13,7 & 913 & 86,3 \\
\hline TOTAL & 2.645 & 29,9 & 6.209 & 70,1 \\
\hline $\begin{array}{c}\text { Indivíduos vivendo em domicílios sem } \\
\text { emigrante }\end{array}$ & \multicolumn{4}{|c|}{ Recebe beneficio social? } \\
\hline TIPOLOGIAS DE MOBILIDADE & SIM & $\%$ & NÃO & $\%$ \\
\hline (1) INDIVÍDUOS IMÓVEIS & 3.213 & 30,1 & 7.449 & 69,9 \\
\hline $\begin{array}{l}\text { (2) INDIVÍDUOS QUE PRATICAM } \\
\text { MOBILIDADE COTIDIANA OU SAZONAL }\end{array}$ & 1.425 & 20,8 & 5.418 & 79,2 \\
\hline $\begin{array}{l}\text { (3) INDIVÍDUOS QUE RESIDIRAM EM } \\
\text { OUTRO MUNICÍPIO }\end{array}$ & 5.736 & 38,2 & 9.266 & 61,8 \\
\hline $\begin{array}{c}\text { (4) INDIVÍDUOS QUE RESIDIRAM } \\
\text { EM OUTRO MUNICÍPIO E PRATICAM } \\
\text { MOBILIDADE COTIDIANA OU SAZONAL }\end{array}$ & 2.118 & 29,4 & 5.074 & 70,6 \\
\hline TOTAL & 12.492 & 31,5 & 27.207 & 68,5 \\
\hline
\end{tabular}

Fonte: Survey Seridó Potiguar, 2017.

Especialmente em se tratando dos indivíduos que praticam mobilidade cotidiana ou sazonal, a Tabela 4 mostra diferenças claras nas proporções se compararmos os indivíduos vivendo em domicílios com emigrante $(5,4 \%)$ e sem emigrante $(20,8 \%)$ que possuem algum tipo de benefício social.

Do mesmo modo ocorre para os indivíduos que praticam mobilidade cotidiana ou sazonal e ao mesmo tempo já residiram em outro município e que são beneficiários de programas sociais, com uma proporção maior para aqueles que vivem em domicílios sem emigrante $(29,4 \%)$ em relação aos que vivem em domicílios com emigrante $(13,7 \%)$. Assim, essas constatações indicam que a mobilidade cotidiana ou sazonal pode ser uma alternativa para aqueles que não se moveram permanentemente e que os programas sociais podem ter um papel importante nessa relação.

Apesar de nossos resultados não serem capazes de fazer afirmações sobre causalidade entre ser beneficiário de programas sociais e escolher determinadas formas de mobilidade, deve ser considerada a relevância desses programas para os indivíduos, especialmente para aqueles que não efetuam nenhuma das formas de mobilidade analisadas ou apenas praticam mobilidade em intervalos de tempo e distâncias menores, ao fornecer um incremento na renda domicliar.

Outros estudos, entretanto, têm apontado que os programas de transferência de renda contribuíram para que a população permanecesse na região (ARAÚJO, 2012; GAMA, 2012), embora tenham desconsiderado o papel secundário desses programas sociais em sua maior influência sobre o processo migratório, permitindo tanto a migração do beneficiário quanto de outro indivíduo ao subsidiar a migração de pelo menos um morador do domicílio (CORREIA; OJIMA, 2018); e sobre as categorias de mobilidade que tratamos nesse trabalho.

Todavia, tais estratégias políticas devem ser mais focalizadas para que esses processos maximizem as oportunidades de escolhas. Isso é uma tarefa difícil, especialmente devido à dependência dos 
municípios do semiárido em relação aos recursos federais. A oferta de serviços em áreas urbanas também depende de maior capacidade orçamentária por parte desses municípios. Em mais de $81 \%$ desses municípios a participação de atividades do PIB relativas à administração, saúde e educação e seguridade social representa mais de $1 / 3$ da economia, as quais dependem principalmente das transferências constitucionais como o Fundo de Participação dos Municípios (FPM) (IBGE, 2012; STN, 2016).

\section{CONSIDERAÇÕES FINAIS}

Como observado em outras regiões do mundo, os nossos resultados mostraram que as secas na região do Seridó potiguar, no Nordeste brasileiro, estão mais associadas a formas de mobilidade de curto prazo, como a mobilidade cotidiana ou sazonal. Também encontramos maior participação feminina nessas formas de mobilidade e menos inserção dos indivíduos mais móveis em ocupações agrícolas. Isso mostra a relevância da mobilidade como um mecanismo para acessar capitais via mercado de trabalho e ocupações urbanas.

Desse modo, a mobilidade é resultado de um processo multicausal, cujos efeitos da mudança ambiental interagem com as características individuais, dos domicílios e estruturais. Certamente, há outros fatores que podem afetar a mobilidade e a (i)mobilidade como resposta à seca que não foram objetos de investigação, e que serão em investigação futura; mas os que estão apontados aqui estão baseados na literatura sobre o semiárido como fatores-chave em uma escala domiciliar e, por isso, foram objeto de investigação.

Desse modo, os principais resultados mostraram que os efeitos das mudanças ambientais sobre as respostas de mobilidade e estratégias de adaptação das populações da região semiárida foram indiretos ao afetar os meios de subsistência. Isso pode ser constatado quando observamos que as TLM têm maior impacto sobre a população dos municípios do semiárido em relação aos demais e nossos resultados mostraram que as populações do Seridó, no entanto, responderam majoritariamente que migrariam por motivo de trabalho $(30,7 \%)$ enquanto apenas $18,1 \%$ migrariam por causa da seca.

As respostas adaptativas foram predominantemente de curto prazo, com uma participação feminina girando em torno dos $70 \%$ nos movimentos de curta distância. Os programas de transferência de renda se mostraram importantes para que a mobilidade cotidiana ou sazonal fosse uma alternativa para os que não migraram, especialmente para os indivíduos que vivem em domicílios sem emigrante (20,8\%). Esses dois resultados foram importantes para destacar o papel secundário dos programas de transferência de renda sobre o poder de barganha das decisões de mobilidade e sobre as respostas adaptativas da população do semiárido. Essas relações são necessárias para realçar nosso conhecimento sobre as respostas adaptativas às secas no semiárido, embora ainda conhecemos pouco sobre a atuação desse fenômeno sobre os meios de subsistência das famílias.

Ainda nos deparamos, desse modo, com um dos problemas mais recorrentes nos estudos de População e Ambiente: qual a escala temporal e espacial de atuação da seca? A seca tem sido presente no discurso da população mesmo nos períodos chuvosos, seja como uma projeção que provoca incertezas sobre o futuro e modifica as expectativas adaptativas das famílias ou como uma materialização fantasmagórica de um fenômeno social. A seca, sem dúvida alguma, contribui para reiterar processos de vulnerabilidade já existentes, principalmente no que concerne a populações mais carentes e expostas a eventos extremos como é o caso dos mais de 22 milhões de habitantes do semiárido brasileiro. Não obstante, nas últimas décadas as políticas de proteção social focalizadas na Região Nordeste acarretaram mudanças que precisam ser melhor discutidas, em especial no contexto das mudanças climáticas.

Há uma necessidade de criação de políticas em nível local, porém, articuladas às demais escalas, e que considerem as particularidades do semiárido. Tais políticas devem considerar tanto medidas de mitigação como de adaptação da população aos processos já em curso, tal como os períodos de estiagem prolongados. No Brasil como um todo ainda existem falhas nas políticas ao passo que as estratégias de enfrentamento são mais focadas em mitigação e menos em adaptação, e que as singularidades das diversas escalas não são consideradas. 
No caso particular do semiárido brasileiro, os programas sociais têm desempenhado um papel relevante na vida dos indivíduos que permanecem na região, inclusive aqueles que efetuam formas de mobilidade de curta distância. A escolha por essas formas de mobilidade se apresenta mais como um sinônimo de resistência do povo do que uma estratégia de adaptação. Políticas públicas mais específicas demandam desafios para as instituições no que diz respeito à capacidade orçamentária dos municípios e exigem o esforço e a cooperação de gestores de órgãos públicos.

Esses desafios dependem, inclusive, da consolidação de uma agenda de pesquisa que dê conta da complexidade das relações entre as secas, as condições de vida e as estratégias de sobrevivência da população do semiárido brasileiro como um todo. Além disso, o papel dos programas sociais como o Bolsa Família e das transferências constitucionais para o semiárido precisa ser melhor compreendido. Essas transferências podem causar dependência por parte dos municípios, especialmente nos períodos de estiagem, mas também podem equalizar situações de vulnerabilidade social.

\section{NOTAS}

${ }^{1}$ Tratamos aqui como urbanização a proporção de pessoas vivendo na zona urbana.

${ }^{2} \mathrm{~A}$ Razão de Sexo refere-se ao número de homens para cada grupo de 100 mulheres.

\section{AGRADECIMENTOS}

Os autores agradecem o suporte financeiro à pesquisa que originou este artigo, por meio da Rede Brasileira de Pesquisas em Mudanças Climáticas (Rede Clima, Finep/MCTI) e do Conselho Nacional de Desenvolvimento Científico e Tecnológico (CNPq), 447688/2014-6 e 306567/2016-4. Também agradecemos à agência de fomento Coordenação de Aperfeiçoamento de Pessoal de Nível Superior (Capes/Proex) a bolsa de doutorado.

\section{REFERÊNCIAS}

AB'SABER, A. N. Sertões e sertanejos: uma geografia humana sofrida. Estudos Avançados, IEA/USP, v. 13, n. 36, p. 7-59, 1999.

ADGER, W. N. et al. Focus on environmental risks and migration: causes and consequences. Environ. Res. Lett., v. 10, n. 1, 060201, 2015.

ALTO COMISSARIADO DAS NAÇÕES UNIDAS PARA OS REFUGIADOS. Working with the Internally Displaced. 2012. UNHCR Global Appeal 2012-2013.

ARAÚJO, T. B. Economia do semiárido nordestino: a crise como oportunidade. Revista Coletiva, v. 16, n. 1, 2012.

BARBIERI, A. F. Mudanças climáticas, mobilidade populacional e cenários de vulnerabilidade para o Brasil. Rev. Interdiscip. Mobil. Hum., v. 36, p. 95-112, 2011.

BARBIERI, A. F. et al. Climate change and population migration in Brazil's Northeast: scenarios for 2025-2050. Popul. Environ., v. 31, p. 344-370, 2010.

BARBIERI, A. et al. Mobility as a livelihood adaptation in the Brazilian semi-árid. In: CONFERENCE MIGRATIONS, ENVIRONMENT AND CLIMATE: WHAT RISKS INEQUALITIES? 1., 2018, Paris. Annals of... Paris, France: Ined, 2018.

BARDSLEY, D. K.; HUGO, G. J. Migration and climate change: examining thresholds of change to guide effective adaptation decision-making. Popul. Environ., v. 32, n. 2-3, p. 238-262, 2010.

BILSBORROW, R. E. Population pressure and agricultural development in developing countries: a conceptual framework and recent evidence. World Development, New York, v. 15, n. 2, p. 183-203, 1987. 
BLACK, R. et al. Climate change: migration as adaptation. Nature, v. 478, n. 7370, p. 477-479, 2011.

BRASIL. Nova delimitação do Semiárido Brasileiro. Brasília/DF: Ministério da Integração Nacional, 2005.

BURSZTYN, M. O poder dos donos, 20 anos depois... ou a incrível e triste história das populações desfavorecidas e suas elites desalmadas. Raízes, v. 22, n. 1, p. 73-79, jan./jun. 2003.

CASTRO, J. Geografia da fome: o dilema brasileiro - pão ou aço. 14. ed. Rio de Janeiro: Civilização Brasileira, 2001.

CORREIA, I. A.; OJIMA, R. Emigração e imobilidade no Nordeste Brasileiro: adaptação ou resistência? Revista de Desenvolvimento Econômico, v. 3, p. 175-192, 2017.

Composição demográfica domiciliar e (i)mobilidade no Seridó Potiguar: vulnerabilidade à seca e estratégias domiciliares no sertão nordestino. Territórios e Fronteiras, v. 11, p. 199-223, 2018.

CUNHA, J. M. P. Migração e urbanização no Brasil: alguns desafios metodológicos para análise. São Paulo em Perspectiva, v. 19, p. 3-20, 2005.

DENALDI, R. et al. Urbanização de favelas na região do ABC no âmbito do Programa de Aceleração do Crescimento - Urbanização de Assentamentos Precários. Cad. Metrop., v. 18, p. 101-118, 2016.

DENTON, F. Climate change vulnerability, impacts and adaptation: why does gender matter? Gender and Development, v. 10, n. 2, p. 10-20, 2002.

DE NYS, E.; ENGLE, N. L.; MAGALHÃES, A. R. (Org.). Secas no Brasil: política e gestão proativas. Brasília/DF: Centro de Gestão e Estudos Estratégicos (CGEE); Banco Mundial, 2016.

FURTADO, C. A operação Nordeste. Rio de Janeiro: Ministério da Educação e Cultura, Instituto Superior de Estudos Brasileiros, 1959.

FUSCO, W.; DUARTE, R. Regiões metropolitanas do Nordeste: origens, destinos e retorno dos migrantes. In: ENCONTRO NACIONAL SOBRE MIGRAÇÕES, 17., 2010, Caxambu. Anais... Caxambu/MG: Abep, 2010.

GAMA, L. C. D. O Programa Bolsa Família pode influenciar a decisão por migrar? Uma análise para o estado de Minas Gerais. In: SEMINÁRIO SOBRE A ECONOMIA MINEIRA, 15., 2012, Diamantina. Anais... Diamantina/MG, 2012.

GIBSON, C.; OSTROM, E.; AHN, T. K. The concept of scale and the human dimensions of global change: a survey. Ecological Economics, v. 32, p. 217-239, 2000.

GONÇALVES, A. J. Migrações internas: evoluções e desafios. Estudos Avançados, v. 15, n. 43. São Paulo, 2001.

GRAY, C.; MUELLER, V. Drought and population mobility in rural Ethiopia. World Development, v. 40, n. 1, p. 134145, 2012.

HANDMER, J. W.; DOVERS, S.; DOWNING, T. E. Societal vulnerability to climate change and variability. Mitigation and Adaptation Strategies for Global Change, v. 4, n. 3/4, p. 267-281, 1999.

HENRY, S.; SCHOUMAKER, B.; BEAUCHEMIN, C. The impact of rainfall on the first out-migration: a multi-level event-history analysis in Burkina Faso. Popul. Environ., v. 25, n. 5, p. 423-460, 2004.

HUGO, G. Environmental Concerns and International Migration. Int. Migr. Rev., v. 30, n. 1, 1996, p. $105-31$.

INSTITUTO BRASILEIRO DE GEOGRAFIA E ESTATÍSTICA. Censo Demográfico do Brasil. Rio de Janeiro: IBGE, 2010.

INSTITUTO BRASILEIRO DE GEOGRAFIA E ESTATístICA. Produto Interno Bruto dos Municípios 2012. Rio de Janeiro: IBGE, 2012.

INTERGOVERNMENTAL PANEL ON CLIMATE CHANGE. In: FIELD, C. B. et al. (Ed.). Managing the risks of extreme events and disasters to advance climate change adaptation: a special report of working groups I and II of the Intergovernmental Panel on Climate Change. New York, NY: Cambridge University Press, 2012. 
INTERGOVERNMENTAL PANEL ON CLIMATE CHANGE. Summary for Policymakers. In: STOCKER, T. F. et al. (Ed.). Climate change 2013: the physical science basis: contribution of working group 1 to the fifth assessment report of the intergovernmental panel on climate change (p. 1-30). Cambridge, United Kingdom: Cambridge University Press, 2013.

INTERGOVERNMENTAL PANEL ON CLIMATE CHANGE. Fifth Assessment Report (AR5). Climate Change 2014: impacts, adaptation, and vulnerability. Part B: Regional Aspects. Chapter 24: Asia, p.1355, 2014.

KOUBI, V.; STOLL, S.; SPILKER, G. Perceptions of environmental change and migration decisions. Climatic Change, v. 138, p. 439-451, 2016.

LEE, E. Uma teoria sobre a migração. In: MOURA, H. A. (Org.). Migração interna, textos selecionados. Fortaleza: BNB/Etene, 1980, p. 89-114, 722p., 1966.

LOBO, C. F. F. et al. Expanded commuting in the metropolitan region of Belo Horizonte: evidence for reverse commuting. R. Bras. Est. Pop., v. 32, p. 219-233, 2015.

MARTINE, G. A redistribuição espacial da população brasileira durante a década de 1980. Texto para Discussão. Brasília: Ipea, 1994.

MORTON, A.; BONCOUR, P.; LACZKO, F. Human security policy changes. Forced Migr. Rev., v. 31, p. 5-7, 2008.

MYERS, N. Environmental refugees: a growing phenomenon of the twenty-first century. Philos. Trans. R. Soc., v. 357, n.1420, p. 609-613, 2002.

NAWROTZKI, R. J.; DEWAARD, J. Climate shocks and the timing of migration from Mexico. Popul. Environ., v. 38, p. 72-100, 2016.

OJIMA, R. Impacto dos programas de transferência de renda sobre a dinâmica populacional e redução da pobreza no Semiárido Setentrional. Relatório Final. Ministério do Desenvolvimento Social e Combate à Fome (MDS). Conselho Nacional de Desenvolvimento Científico e Tecnológico (CNPq). Edital MCTI-CNPq/MDS-SAGI noㅡ 24/2013. 2015.

OJIMA, R.; MARTINE, G. Resgates sobre população e ambiente: breve análise da dinâmica demográfica e a urbanização nos biomas brasileiros. Ideias, v. 1, p. 55-70, 2012.

RAVENSTEIN, E. G. The laws of migration. Journal of the Royal Statistical Society, v. 52, n. 2, p. 241-305, 1889.

RIBOT, J. C. Introduction. Climate variability, climate change and vulnerability: moving forward by looking back. In: RIBOT, J. C.; MAGALHÃES, A. R.; PANAGIDES, S. S. (Comps.). Climate variability, climate change and social vulnerability in the semi-arid tropics. Reino Unido e Nova lorque: Cambridge University Press, 1996. p. 1-13.

SECRETARIA DO TESOURO NACIONAL. Fundo de Participação dos Municípios. Brasília/DF, 2016. Disponível em: <http://sisweb.tesouro.gov.br/apex/f?p=2600:1::MOSTRA:NO:RP::>. Acesso em: 1 set. 2017.

SHERBININ, A. et al. Rural household demographics, livelihoods and the environment. Glob. Environ. Change, v. 18, n. 1, p. 38-53, 2008.

SILVA, L. S.; TRAVASSOS, L. Problemas ambientais urbanos: desafios para a elaboração de políticas públicas integradas. Cad. Metrop., v. 19, p. 27-47, 2008.

SIVAKUMAR, M. V. K.; DAS, H. P.; BRUNINI, O. Impacts of present and future climate variability and change on agriculture and forestry in the arid and semi-arid tropics. Climatic Change, v. 70, n. 1, p. 31-72, 2005.

STARK, O.; BLOOM, D. E. The new economics of labor migration. Am. Econ. Rev., v. 75, n. 2, p. 173-178, 1985.

VANWEY, L. K.; GUEDES, G. R.; D’ANTONA, A. O. Out-migration and land-use change in agricultural frontiers: insights from Altamira settlement project. Popul. Environ., v. 34, n. 1, p. 44-68, 2012. 